\title{
PREVALENCE OF HEADACHE IN CHILDREN OF A SCHOOL FROM CURITIBA, BRAZIL, COMPARING DATA OBTAINED FROM CHILDREN AND PARENTS
}

\author{
SÉRGIO ANTONIUK*, MARCELO FELIPE KOZAK**, LEANDRO MICHELON**, \\ MÁRIO RODRIGUES MONTEMÓR NETTO**
}

\begin{abstract}
This study analyzed prevalence, frequency and cause of headache among 460 children ranging from 10 to 14 years-old from a Brazilian school. A questionnaire was handed both to children and parents to know if there would be differences among children and parental reports. The lifetime prevalence of headache was $93.5 \%$ (children reports) and $93.3 \%$ (parental reports). The last year prevalence was $90 \%$ (children) and $89.8 \%$ (parents). Headache episodes were frequent in $17.6 \%$ (children) and $18.5 \%$ (parents). The most often reported cause was "flu" ( $39.1 \%$ by children, and $46.7 \%$ by parents). This study demonstrated that the prevalence of headache in children is high; moreover, there were noted few differences between data obtained from children and parents. So, we could say that when the objective of a epidemiologic study is to determine the prevalence of headache in children, both children and parental reports may be used.
\end{abstract}

KEY WORDS: headache, schoolchildren, epidemiology.

Prevalência de cefaléia em crianças de uma escola de Curitiba, Brasil, comparando dados obtidos de crianças e pais

RESUMO - Este estudo analisou prevalência, frequência e motivo da cefaléia entre 460 crianças de 10 a 14 anos de uma escola brasileira. Com a finalidade de saber se haveria diferenças obtendo informações das crianças ou dos pais, um questionário foi entregue a ambos. A prevalência durante a vida foi 93,5\% (relatos das crianças) e 93,3\% (relatos dos pais). A prevalência nos últimos 12 meses foi $90 \%$ (crianças) e 89,8\% (pais). As cefaléias foram frequentes em 17,6\% (crianças) e 18,5\% (pais). O motivo mais citado foi "gripe" (39,1\% pelas crianças e $46,7 \%$ pelos pais). Esse estudo demonstrou ser alta a prevalência de cefaléia entre crianças; além disso, poucas diferenças entre dados obtidos de crianças e pais foram observadas. Dessa forma, poderíamos dizer que quando o objetivo de um estudo epidemiológico é determinar a prevalência de cefaléia em crianças, tanto os relatos das crianças como os dos pais podem ser utilizados.

PALAVRAS-CHAVE: cefaléia, escolares, epidemiologia.

Headache is one of the most common causes of consultation to the pediatrician ${ }^{1}$. Headache disorders are a "social" phenomenon of considerable dimensions because they may interfere in the patient's life and in his/her family's quotidian, principally when they are severe and frequent, causing considerable suffering as well as decreased or abolished working capacity ${ }^{2}$.

Generally, headache is a common and benign symptom among schoolchildren and adolescents; however, it is less frequent and seems to be related to more severe pathologies in younger children ${ }^{3}$. Most of the patients seen for headache in a walk-in clinic did not have a serious symptomatic cause; they have, instead of, an acute febrile illness associated with their complaint ${ }^{4}$.

Estudo realizado junto ao Departamento de Pediatria do Hospital de Clínicas (HC) da Universidade Federal do Paraná (UFPR), Curitiba, Brasil: *Professor Assistente de Neuropediatria do Departamento de Pediatria do HC/ UFPR; **Acadêmico do $6^{\circ}$ ano do curso de Medicina, Setor de Ciências da Saúde, UFPR. Aceite: 11-agosto-1998. 
In the life of a child, school represents an important stress factor similar to that of working life in adulthood ${ }^{5}$. To approach puberty, that occurs at an age of about 13 years, is also a stress factor. In Brazil, due to its particular social and economic characteristics, the uncertainty in the life of many students, who sometimes have to decide between to pursue their academic lives or to look for a job to help their familial income, is another stress factor. All these factors increase the incidence of psychosomatic disorders and symptoms like headache ${ }^{6}$.

The classification and epidemiology of headache disorders in pediatrics is well worth studying for some reasons. First, epidemiologic studies determine the scope and distribution of the public health problems related to headache. Second, these studies will help identify the risk factors for various pediatric headache disorders. Third, longitudinal studies can help define the natural history of headache disorders in childhood. Fourth, these studies can help determine the level of health care utilization and define the health care needs. And, finally, epidemiologic studies can facilitate a clinical standardization applicable both to children and adults ${ }^{7,8}$.

The objectives of the present study were (i) to determine the prevalence of headache in children of a school from the city of Curitiba (south of Brazil) and (ii) to make an comparative analysis among information obtained from children and parents. The two main reasons that let us to initiate this study were: first, the fact that headache is one of the most important complaints involuntarily mentioned by children and, second, the discrepancies often found between child and parental report ${ }^{9}$.

\section{METHODS}

Subjects: 755 children ranging from 10 to 14 years of age matriculated from the 5th to the 8th year of a public school from the city of Curitiba, PR, Brazil, during the 1st semester of 1997.

Material: a questionnaire with 4 questions: 1. Did you ever have headache in your life? (yes or no); 2. Did you have headache in the last year? (yes or no); 3 . What was the frequency of headache attacks in the last year? (rarely, at least once a month, more than once a week, almost every day, "other" or "don't know"); 4. What was the cause of the headache? (a hit in head, flu, nervousness, difficulty to see, migraine, sinusitis, allergy, "other" and/or "don't know"). The same model of questionnaire was delivered to parents answer about their children. Data as name, age and sex were also obtained.

Methods: first of all, we obtained the permission from the directory of the school to perform this study. After, we went to the classrooms and explained to teachers and students we were doing a research about headache. Then, the questions were read aloud and the children were directed to make questions if there was any doubt. Finally, the questionnaires were distributed to the students, who had 10 minutes to fulfill them. The notation of more than one response in the last question was allowed. The questionnaires were collected after all students had answered them. After this first part, we distributed the questionnaires destined to the children's parents, which ought to be returned in two days, when we would collect them. The distribution of frequency and cause of headache was analyzed only in the questionnaires where there was complaint of headache in the last year. To compare the data obtained from children and parents, only the questionnaires of the students whose parents answered them were analyzed. To evaluate the results, we used a descriptive method, as guided by the Statistic Laboratory of the Universidade Federal do Paraná, Brazil.

\section{RESULTS}

A total of 755 questionnaires were distributed to students and parents. Of the 755 questionnaires handed to the parents, 555 (73.5\%) were answered by them. However, only 460 (60.9\%) could be evaluated. The others (95) were excluded because they were not well fulfilled by children and/or parents. Of the 460 students evaluated, 274 (59.6\%) were girls and $186(40.4 \%)$ boys. The mean age was 11.9 years (Table 1$)$.

\section{Questionnaires from children}

The lifetime prevalence of headache was $93.5 \%$ (female $=94.5 \%$; male $=91.9 \%$ ). Considering age and gender, it was observed that all the girls of 14 years of age had already presented headache, and that the boys of 13 years of age presented the lesser prevalence (Table 2). 
Table 1. Distribution of the subjects by age and gender.

\begin{tabular}{lccccc}
\hline Age & 10 & 11 & 12 & 13 & 14 \\
\hline Female & 32 & 65 & 88 & 60 & 29 \\
Male & 25 & 43 & 56 & 37 & 25 \\
Total & $57(12.4 \%)$ & $108(23.5 \%)$ & $144(31.3 \%)$ & $97(21.1 \%)$ & $54(11.7 \%)$ \\
\hline
\end{tabular}

Table 2. Lifetime prevalence of headache by age, gender and informant.

\begin{tabular}{|c|c|c|c|c|c|c|c|c|c|c|}
\hline \multirow{2}{*}{$\begin{array}{l}\text { Age } \\
\text { Informant }\end{array}$} & \multicolumn{2}{|c|}{10} & \multicolumn{2}{|c|}{11} & \multicolumn{2}{|c|}{12} & \multicolumn{2}{|c|}{13} & \multicolumn{2}{|c|}{14} \\
\hline & $\mathrm{C}$ & $\mathrm{P}$ & $\mathrm{C}$ & $\mathrm{P}$ & $\mathrm{C}$ & $\mathrm{P}$ & $\mathrm{C}$ & $\mathrm{P}$ & $\mathrm{C}$ & $\mathrm{P}$ \\
\hline Female & 93.7 & 87.5 & 98.5 & 98.5 & 90.9 & 90.9 & 93.3 & 95 & 100 & 100 \\
\hline Male & 92 & 92 & 95.4 & 88.4 & 92.3 & 92.3 & 86.5 & 89.2 & 92 & 100 \\
\hline Total & 93 & 89.5 & 97.2 & 94.4 & 91.7 & 91.7 & 90.7 & 92.8 & 96.3 & 100 \\
\hline
\end{tabular}

C, children; P, parents.

Table 3. Last year prevalence of headache by age, gender and informant.

\begin{tabular}{lcccccccccc}
\hline Age & \multicolumn{2}{c}{10} & \multicolumn{2}{c}{11} & \multicolumn{2}{c}{12} & \multicolumn{2}{c}{13} & \multicolumn{2}{c}{14} \\
Informant & $\mathrm{C}$ & $\mathrm{P}$ & $\mathrm{C}$ & $\mathrm{P}$ & $\mathrm{C}$ & $\mathrm{P}$ & $\mathrm{C}$ & $\mathrm{P}$ & $\mathrm{C}$ & $\mathrm{P}$ \\
\hline Female & 87.5 & 78.1 & 96.9 & 96.9 & 89.8 & 89.8 & 91.7 & 91.7 & 100 & 100 \\
Male & 84 & 92 & 90.7 & 88.4 & 87.5 & 87.5 & 78.4 & 83.8 & 88 & 84 \\
Total & 86 & 84.2 & 94.4 & 93.5 & 88.9 & 88.9 & 86.6 & 88.7 & 94.4 & 92.6 \\
\hline
\end{tabular}

C, children; P, parents.

The last year prevalence of headache was $90 \%$ (female $=92.7 \%$; male $=86 \%$ ). Considering age and gender, it was observed that all the girls of 14 years of age had headache in the last year, and that the boys of 13 years of age presented the lesser prevalence (Table 3 ).

Approximately $40 \%$ of the questionnaires revealed a rare frequency of headache episodes. One hundred and eighty-eight students $(188=45.4 \%)$ had at least one episode a month; 73 of them $(17.6 \%)$ had headache more than once a week or almost every day. Comparing gender and frequency of headache, it was noted that male students had less frequent episodes. In accord to age, it was observed that the oldest children had less frequent episodes of headache (Table 4).

The most common causes of headache were "flu" (39.1\%), and "nervousness" (22.9\%). Approximately $24 \%$ of the students did not know the provoking factor of the headache (Table 5). It is important to emphasize that of those children who had frequent headaches ("more than once a week" or "almost every day"), 19,2\% did not know the reason of the pain, that represents $3 \%$ of the sample.

\section{Questionnaires from parents}

The lifetime prevalence of headache was $93.3 \%$ (female $=94.2 \%$; male $=91.9 \%$ ). Considering age and gender, it was observed that all the girls and boys of 14 years-old had already presented headache, and that the boys of 10 years of age presented the lesser prevalence (Table 2).

The last year prevalence of headache was $89,8 \%$ (female $=91.6 \%$; male $=87.1 \%$ ). Considering age and gender, it was observed that all the girls of 14 years of age had headache in the last year, and that the boys of 10 years of age presented the lesser prevalence (Table 3 ). 
Table 4. Distribution of headache frequency according to age, gender and informant.

\begin{tabular}{|c|c|c|c|c|c|c|c|c|c|c|c|c|c|c|}
\hline \multirow{2}{*}{$\begin{array}{l}\text { Age } \\
\text { Gender }\end{array}$} & & \multicolumn{2}{|c|}{10} & \multicolumn{2}{|c|}{11} & \multicolumn{2}{|c|}{12} & \multicolumn{2}{|c|}{13} & \multicolumn{2}{|c|}{14} & \multicolumn{3}{|c|}{ Total } \\
\hline & & F & M & F & M & F & M & F & M & F & M & F & $\mathrm{M}$ & $\mathrm{F}+\mathrm{M}$ \\
\hline \multirow[t]{2}{*}{ Rarely } & $\mathrm{C}$ & $\begin{array}{c}7 \\
(21.9)\end{array}$ & $\begin{array}{c}10 \\
(40)\end{array}$ & $\begin{array}{c}21 \\
(32.3)\end{array}$ & $\begin{array}{c}16 \\
(37.2)\end{array}$ & $\begin{array}{c}26 \\
(29.5)\end{array}$ & $\begin{array}{c}21 \\
(37.5)\end{array}$ & $\begin{array}{c}21 \\
(35)\end{array}$ & $\begin{array}{c}13 \\
(35.1)\end{array}$ & $\begin{array}{c}15 \\
(51.7)\end{array}$ & $\begin{array}{c}14 \\
(56)\end{array}$ & $\begin{array}{c}90 \\
(35.4)\end{array}$ & $\begin{array}{c}74 \\
(46.2)\end{array}$ & $\begin{array}{c}164 \\
(39.6)\end{array}$ \\
\hline & $\mathrm{P}$ & $\begin{array}{c}13 \\
(40.6)\end{array}$ & $\begin{array}{c}10 \\
(40)\end{array}$ & $\begin{array}{c}27 \\
(41.5)\end{array}$ & $\begin{array}{c}22 \\
(51.2)\end{array}$ & $\begin{array}{c}41 \\
(46.6)\end{array}$ & $\begin{array}{c}24 \\
(42.8)\end{array}$ & $\begin{array}{c}19 \\
(31.7)\end{array}$ & $\begin{array}{c}17 \\
(45.9)\end{array}$ & $\begin{array}{c}14 \\
(48.3)\end{array}$ & $\begin{array}{c}11 \\
(44)\end{array}$ & $\begin{array}{c}114 \\
(45.4)\end{array}$ & $\begin{array}{c}84 \\
(51.8)\end{array}$ & $\begin{array}{c}198 \\
(47.9)\end{array}$ \\
\hline
\end{tabular}

$\begin{array}{lllllllllllllll}\text { At least } & \mathrm{C} & 8 & 4 & 16 & 11 & 25 & 14 & 17 & 9 & 8 & 3 & 74 & 41 & 115\end{array}$

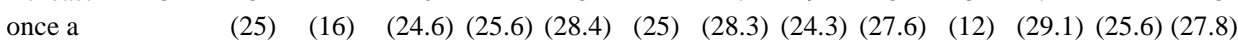

$\begin{array}{lllllllllllllll}\text { month } & \mathrm{P} & 5 & 6 & 14 & 9 & 18 & 13 & 16 & 7 & 7 & 6 & 60 & 41 & 101\end{array}$

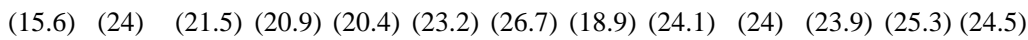

$\begin{array}{lcccccccccccccc}\text { More } & \mathrm{C} & 3 & 2 & 8 & 1 & 8 & 3 & 8 & 3 & 3 & 2 & 30 & 11 & 41 \\ \text { than once } & & (9.4) & (8) & (12.3) & (2.3) & (9.1) & (5.4) & (13.3) & (8.1) & (10.3) & (8) & (11.8) & (6.9) & (9.9) \\ \text { a week } & \mathrm{P} & 2 & 3 & 10 & 3 & 7 & 3 & 13 & 3 & 5 & 2 & 37 & 14 & 51 \\ & & (6.2) & (12) & (15.4) & (7) & (8) & (5.4) & (21.7) & (8.1) & (17.2) & (8) & (14.7) & (8.6) & (12.4)\end{array}$

$\begin{array}{lcccccccccccccc}\text { Almost } & \mathrm{C} & 6 & 1 & 4 & 1 & 6 & 5 & 5 & 1 & 3 & 2 & 24 & 8 & 32 \\ \text { every } & & (18.8) & (4) & (6.2) & (2.3) & (6.8) & (8.9) & (8.3) & (2.7) & (10.3) & (8) & (9.4) & (5) & (7.7) \\ \text { day } & \mathrm{P} & 4 & 0 & 7 & 1 & 6 & 2 & 4 & 0 & 2 & 2 & 23 & 2 & 25 \\ & & (12.5) & & (10.8) & (2.3) & (6.8) & (3.6) & (6.7) & & (6.9) & (8) & (9.1) & (1.2) & (6.1)\end{array}$

\begin{tabular}{lcccccccccccccc} 
"Don't & $\mathrm{C}$ & 3 & 4 & 11 & 10 & 14 & 5 & 4 & 3 & 2 & 1 & 34 & 23 & 57 \\
Know" & & $(9.4)$ & $(16)$ & $(16.9)$ & $(23.3)$ & $(15.9)$ & $(8.9)$ & $(6.7)$ & $(8.1)$ & $(6.9)$ & $(4)$ & $(13.4)$ & $(14.4)(13.8)$ \\
& $\mathrm{P}$ & 0 & 2 & 7 & 1 & 3 & 4 & 3 & 2 & 1 & 0 & 14 & 9 & 23 \\
& & & $(8)$ & $(10.8)$ & $(2.3)$ & $(3.4)$ & $(7.1)$ & $(5)$ & $(5.4)$ & $(3.4)$ & & $(5.6)$ & $(5.6)$ & $(5.6)$ \\
\hline
\end{tabular}

C, children; P, parents.

Approximately $48 \%$ of the questionnaires revealed a rare frequency of headache episodes. One hundred and seventy-seven $(177=42.8 \%)$ had at least one episode a month; 76 of them $(18.4 \%)$ had headache more than once a week or almost every day. Comparing gender and frequency of headache, it was observed that boys had less frequent episodes: 60 girls (23.9\%) whom had headache in the last year presented this symptom more than once a week and/or almost every day; among boys, this index was $9.8 \%$ (16 students) (Table 4).

The most common causes of headache were "flu" (46.7\%), and "nervousness" (20.6\%). Approximately $19 \%$ of the parents did not know the provoking factor of the headache (Table 5). It is important to emphasize that of those children who had frequent headaches ("more than once a week" or "almost every day"), $21 \%$ did not know the reason of the pain, that represents $3,5 \%$ of the sample.

\section{Similarity between the answers comparing children and parental reports}

The similarity among the answers of the first question was $96.7 \%$. In the second question, the similarity was $96.3 \%$. In the third question, this index was $40.9 \%$ and, finally, it was $29.6 \%$ in the fourth question. The answers were completely equal in 167 children (36.3\%). 
Table 5. Distribution of headache cause by age, gender and informat.

\begin{tabular}{|c|c|c|c|c|c|c|c|c|c|c|c|c|c|c|}
\hline \multirow{2}{*}{$\begin{array}{l}\text { Age } \\
\text { Gender }\end{array}$} & & \multicolumn{2}{|c|}{10} & \multicolumn{2}{|c|}{11} & \multicolumn{2}{|c|}{12} & \multicolumn{2}{|c|}{13} & \multicolumn{2}{|c|}{14} & \multicolumn{3}{|c|}{ Total } \\
\hline & & $\mathrm{F}$ & M & $\mathrm{F}$ & M & $\mathrm{F}$ & $\mathrm{M}$ & $\mathrm{F}$ & M & $\mathrm{F}$ & M & $\mathrm{F}$ & M & $F+M$ \\
\hline $\begin{array}{l}\text { Hit } \\
\text { in }\end{array}$ & $\mathrm{C}$ & 0 & $\begin{array}{c}2 \\
(8)\end{array}$ & $\begin{array}{c}5 \\
(7.7)\end{array}$ & $\begin{array}{c}2 \\
(4.6)\end{array}$ & $\begin{array}{c}4 \\
(4.5)\end{array}$ & $\begin{array}{c}6 \\
(10.7)\end{array}$ & $\begin{array}{c}4 \\
(6.7)\end{array}$ & $\begin{array}{c}1 \\
(2.7)\end{array}$ & $\begin{array}{c}2 \\
(6.9)\end{array}$ & $\begin{array}{c}2 \\
(8)\end{array}$ & $\begin{array}{c}15 \\
(5.9)\end{array}$ & $\begin{array}{c}14 \\
(8.8)\end{array}$ & $\begin{array}{l}29 \\
(7)\end{array}$ \\
\hline head & $\mathrm{P}$ & 0 & 0 & $\begin{array}{c}2 \\
(3.1)\end{array}$ & $\begin{array}{c}1 \\
(2.3)\end{array}$ & $\begin{array}{c}5 \\
(5.7)\end{array}$ & $\begin{array}{c}3 \\
(5.4)\end{array}$ & $\begin{array}{c}4 \\
(6.7)\end{array}$ & 0 & $\begin{array}{c}2 \\
(6.9)\end{array}$ & $\begin{array}{c}3 \\
(12)\end{array}$ & $\begin{array}{c}13 \\
(5.2)\end{array}$ & $\begin{array}{c}7 \\
(4.3)\end{array}$ & $\begin{array}{c}20 \\
(4.8)\end{array}$ \\
\hline Flu & $\mathrm{C}$ & $\begin{array}{c}9 \\
(28.1)\end{array}$ & $\begin{array}{c}7 \\
(28)\end{array}$ & $\begin{array}{c}27 \\
(41.5)\end{array}$ & $\begin{array}{c}19 \\
(44.2)\end{array}$ & $\begin{array}{c}28 \\
(31.8)\end{array}$ & $\begin{array}{c}20 \\
(35.7)\end{array}$ & $\begin{array}{c}19 \\
(31.7)\end{array}$ & $\begin{array}{c}7 \\
(18.9)\end{array}$ & $\begin{array}{c}13 \\
(44.8)\end{array}$ & $\begin{array}{c}13 \\
(52)\end{array}$ & $\begin{array}{c}96 \\
(37.8)\end{array}$ & $\begin{array}{c}66 \\
(41.2)\end{array}$ & $\begin{array}{c}162 \\
(39.1)\end{array}$ \\
\hline & $\mathrm{P}$ & $\begin{array}{c}11 \\
(34.4)\end{array}$ & $\begin{array}{c}8 \\
(32)\end{array}$ & $\begin{array}{c}30 \\
(46.2)\end{array}$ & $\begin{array}{c}24 \\
(55.8)\end{array}$ & $\begin{array}{c}39 \\
(44.3)\end{array}$ & $\begin{array}{c}23 \\
(41.1)\end{array}$ & $\begin{array}{c}21 \\
(35)\end{array}$ & $\begin{array}{c}10 \\
(27)\end{array}$ & $\begin{array}{c}14 \\
(48.3)\end{array}$ & $\begin{array}{c}13 \\
(52)\end{array}$ & $\begin{array}{c}115 \\
(45.8)\end{array}$ & $\begin{array}{c}78 \\
(48.1)\end{array}$ & $\begin{array}{c}193 \\
(46.7)\end{array}$ \\
\hline $\begin{array}{l}\text { Nervous- } \\
\text { ness }\end{array}$ & $\mathrm{C}$ & $\begin{array}{c}7 \\
(21.9)\end{array}$ & $\begin{array}{c}3 \\
(12)\end{array}$ & $\begin{array}{c}18 \\
(27.7)\end{array}$ & $\begin{array}{c}9 \\
(20.9)\end{array}$ & $\begin{array}{c}20 \\
(22.7)\end{array}$ & $\begin{array}{c}10 \\
(17.8)\end{array}$ & $\begin{array}{c}11 \\
(18.3)\end{array}$ & $\begin{array}{c}5 \\
(13.5)\end{array}$ & $\begin{array}{c}7 \\
(24.1)\end{array}$ & $\begin{array}{c}5 \\
(20)\end{array}$ & $\begin{array}{c}62 \\
(24.4)\end{array}$ & $\begin{array}{c}33 \\
(20.6)\end{array}$ & $\begin{array}{c}95 \\
(22.9)\end{array}$ \\
\hline & $\mathrm{P}$ & $\begin{array}{c}6 \\
(18.8)\end{array}$ & $\begin{array}{c}3 \\
(12)\end{array}$ & $\begin{array}{c}14 \\
(21.5)\end{array}$ & $\begin{array}{c}6 \\
(14)\end{array}$ & $\begin{array}{c}20 \\
(22.7)\end{array}$ & $\begin{array}{c}8 \\
(14.3)\end{array}$ & $\begin{array}{c}11 \\
(18.3)\end{array}$ & $\begin{array}{c}7 \\
(18.9)\end{array}$ & $\begin{array}{c}6 \\
(20.7)\end{array}$ & $\begin{array}{c}4 \\
(16)\end{array}$ & $\begin{array}{c}57 \\
(22.7)\end{array}$ & $\begin{array}{c}28 \\
(17.3)\end{array}$ & $\begin{array}{c}85 \\
(20.6)\end{array}$ \\
\hline $\begin{array}{l}\text { Difficulty } \\
\text { to }\end{array}$ & $\mathrm{C}$ & $\begin{array}{c}4 \\
(12.5)\end{array}$ & $\begin{array}{c}4 \\
(16)\end{array}$ & $\begin{array}{c}7 \\
(10.8)\end{array}$ & $\begin{array}{c}3 \\
(7)\end{array}$ & $\begin{array}{c}8 \\
(9.1)\end{array}$ & $\begin{array}{c}6 \\
(10.7)\end{array}$ & $\begin{array}{c}6 \\
(10)\end{array}$ & $\begin{array}{c}1 \\
(2.7)\end{array}$ & $\begin{array}{c}2 \\
(6.9)\end{array}$ & 0 & $\begin{array}{c}27 \\
(10.6)\end{array}$ & $\begin{array}{c}14 \\
(8.8)\end{array}$ & $\begin{array}{c}41 \\
(9.9)\end{array}$ \\
\hline see & $\mathrm{P}$ & $\begin{array}{c}6 \\
(18.8)\end{array}$ & $\begin{array}{c}4 \\
(16)\end{array}$ & $\begin{array}{c}9 \\
(13.8)\end{array}$ & $\begin{array}{c}2 \\
(4.6)\end{array}$ & $\begin{array}{c}9 \\
(10.2)\end{array}$ & $\begin{array}{c}7 \\
(12.5)\end{array}$ & $\begin{array}{c}7 \\
(11.7)\end{array}$ & $\begin{array}{c}2 \\
(5.4)\end{array}$ & $\begin{array}{c}4 \\
(13.8)\end{array}$ & $\begin{array}{c}2 \\
(8)\end{array}$ & $\begin{array}{c}35 \\
(13.9)\end{array}$ & $\begin{array}{c}17 \\
(10.5)\end{array}$ & $\begin{array}{c}52 \\
(12.6)\end{array}$ \\
\hline Migraine & $\mathrm{C}$ & $\begin{array}{c}1 \\
(3.1)\end{array}$ & $\begin{array}{c}1 \\
(4)\end{array}$ & $\begin{array}{c}4 \\
(6.2)\end{array}$ & $\begin{array}{c}1 \\
(2.3)\end{array}$ & $\begin{array}{c}3 \\
(3.4)\end{array}$ & $\begin{array}{c}1 \\
(1.8)\end{array}$ & $\begin{array}{c}3 \\
(5)\end{array}$ & 0 & $\begin{array}{c}1 \\
(3.4)\end{array}$ & $\begin{array}{c}1 \\
(4)\end{array}$ & $\begin{array}{c}12 \\
(4.7)\end{array}$ & $\begin{array}{c}4 \\
(2.5)\end{array}$ & $\begin{array}{c}16 \\
(3.9)\end{array}$ \\
\hline & $\mathrm{P}$ & $\begin{array}{c}2 \\
(6.2)\end{array}$ & 0 & $\begin{array}{c}4 \\
(6.2)\end{array}$ & $\begin{array}{c}2 \\
(4.6)\end{array}$ & $\begin{array}{c}4 \\
(4.5)\end{array}$ & $\begin{array}{c}2 \\
(3.6)\end{array}$ & $\begin{array}{c}6 \\
(10)\end{array}$ & $\begin{array}{c}1 \\
(2.7)\end{array}$ & $\begin{array}{c}3 \\
(10.3)\end{array}$ & 0 & $\begin{array}{c}19 \\
(7.6)\end{array}$ & $\begin{array}{c}5 \\
(3.1)\end{array}$ & $\begin{array}{c}24 \\
(5.8)\end{array}$ \\
\hline Sinusitis & $\mathrm{C}$ & $\begin{array}{c}2 \\
(6.2)\end{array}$ & 0 & $\begin{array}{c}4 \\
(6.2)\end{array}$ & 0 & $\begin{array}{c}9 \\
(10.2)\end{array}$ & $\begin{array}{c}4 \\
(7.1)\end{array}$ & $\begin{array}{c}5 \\
(8.3)\end{array}$ & $\begin{array}{c}3 \\
(8.1)\end{array}$ & $\begin{array}{c}2 \\
(6.9)\end{array}$ & $\begin{array}{c}1 \\
(4)\end{array}$ & $\begin{array}{c}22 \\
(8.6)\end{array}$ & $\begin{array}{c}8 \\
(5)\end{array}$ & $\begin{array}{c}30 \\
(7.2)\end{array}$ \\
\hline & $\mathrm{P}$ & $\begin{array}{c}3 \\
(9.4)\end{array}$ & 0 & $\begin{array}{c}3 \\
(4.6)\end{array}$ & $\begin{array}{c}2 \\
(4.6)\end{array}$ & $\begin{array}{c}8 \\
(9.1)\end{array}$ & $\begin{array}{c}2 \\
(3.6)\end{array}$ & $\begin{array}{c}5 \\
(8.3)\end{array}$ & $\begin{array}{c}2 \\
(5.4)\end{array}$ & $\begin{array}{c}4 \\
(13.8)\end{array}$ & $\begin{array}{c}2 \\
(8)\end{array}$ & $\begin{array}{c}23 \\
(9.2)\end{array}$ & $\begin{array}{c}8 \\
(4.9)\end{array}$ & $\begin{array}{c}31 \\
(7.5)\end{array}$ \\
\hline Allergy & $\mathrm{C}$ & $\begin{array}{c}1 \\
(3.1)\end{array}$ & $\begin{array}{c}2 \\
(8)\end{array}$ & $\begin{array}{c}3 \\
(4.6)\end{array}$ & $\begin{array}{c}2 \\
(4.6)\end{array}$ & $\begin{array}{c}2 \\
(2.3)\end{array}$ & $\begin{array}{c}1 \\
(1.8)\end{array}$ & 0 & 0 & $\begin{array}{c}3 \\
(10.3)\end{array}$ & $\begin{array}{c}3 \\
(12)\end{array}$ & $\begin{array}{c}9 \\
(3.5)\end{array}$ & $\begin{array}{c}8 \\
(5)\end{array}$ & $\begin{array}{c}17 \\
(4.1)\end{array}$ \\
\hline & $\mathrm{P}$ & $\begin{array}{c}2 \\
(6.2)\end{array}$ & $\begin{array}{c}2 \\
(8)\end{array}$ & $\begin{array}{c}4 \\
(6.2)\end{array}$ & $\begin{array}{c}5 \\
(11.6)\end{array}$ & $\begin{array}{c}5 \\
(5.7)\end{array}$ & $\begin{array}{c}4 \\
(7.1)\end{array}$ & $\begin{array}{c}4 \\
(6.7)\end{array}$ & $\begin{array}{c}2 \\
(5.4)\end{array}$ & $\begin{array}{c}2 \\
(6.9)\end{array}$ & $\begin{array}{c}4 \\
(16)\end{array}$ & $\begin{array}{c}17 \\
(6.8)\end{array}$ & $\begin{array}{c}17 \\
(10.5)\end{array}$ & $\begin{array}{c}34 \\
(8.2)\end{array}$ \\
\hline $\begin{array}{l}\text { "Don't } \\
\text { Know" }\end{array}$ & $\mathrm{C}$ & $\begin{array}{c}9 \\
(28.1)\end{array}$ & $\begin{array}{c}3 \\
(12)\end{array}$ & $\begin{array}{c}14 \\
(21.5)\end{array}$ & $\begin{array}{c}10 \\
(23.3)\end{array}$ & $\begin{array}{c}17 \\
(19.3)\end{array}$ & $\begin{array}{c}9 \\
(16.1)\end{array}$ & $\begin{array}{c}18 \\
(30)\end{array}$ & $\begin{array}{c}11 \\
(29.7)\end{array}$ & $\begin{array}{c}8 \\
(27.6)\end{array}$ & $\begin{array}{c}2 \\
(8)\end{array}$ & $\begin{array}{c}66 \\
(26)\end{array}$ & $\begin{array}{c}35 \\
(21.9)\end{array}$ & $\begin{array}{c}101 \\
(24.4)\end{array}$ \\
\hline & $\mathrm{P}$ & $\begin{array}{c}4 \\
(12.5)\end{array}$ & $\begin{array}{c}4 \\
(16)\end{array}$ & $\begin{array}{c}15 \\
(23.1)\end{array}$ & $\begin{array}{c}7 \\
(16.3)\end{array}$ & $\begin{array}{c}11 \\
(12.5)\end{array}$ & $\begin{array}{c}8 \\
(14.3)\end{array}$ & $\begin{array}{c}17 \\
(28.3)\end{array}$ & $\begin{array}{c}6 \\
(16.2)\end{array}$ & $\begin{array}{c}3 \\
(10.3)\end{array}$ & $\begin{array}{c}3 \\
(12)\end{array}$ & $\begin{array}{c}50 \\
(19.9)\end{array}$ & $\begin{array}{c}28 \\
(17.3)\end{array}$ & $\begin{array}{c}78 \\
(18.9)\end{array}$ \\
\hline
\end{tabular}

C, children; $\mathrm{P}$, parents.

\section{DISCUSSION}

Headache is a subjective complaint without any laboratory correlate, thus any prevalence assessment must be based exclusively on information given by the subjects ${ }^{2}$. The methodological differences among studies about headaches in children and the lack of standardized case definitions have plagued epidemiological studies of headache and have contributed to the enormous variation in estimates of the prevalence and incidence of primary headache disorders, not allowing systematical comparisons $\mathrm{s}^{2,7,10}$. Another source of error is the discrepancy often found between child and parental 
Table 6. Results of previous studies.

\begin{tabular}{|c|c|c|c|c|c|}
\hline $\begin{array}{l}\text { Author, } \\
\text { Country-year }\end{array}$ & Population & Sample & Age & $\begin{array}{l}\text { Lifetime } \\
\text { prevalence }\end{array}$ & $\begin{array}{l}\text { Last year } \\
\text { prevalence }\end{array}$ \\
\hline $\begin{array}{l}\text { Rasmussen et al. } \\
\text { Denmark - } 1988\end{array}$ & Community & 740 & $25-64$ & $\begin{array}{c}96 \% \\
\text { female }=99 \% \\
\text { male }=93 \%\end{array}$ & - \\
\hline $\begin{array}{l}\text { Silanpää } \\
\text { Finland - } 1983\end{array}$ & $\begin{array}{l}\text { School- } \\
\text { children }\end{array}$ & 2921 & 14 & $69 \%$ & - \\
\hline $\begin{array}{l}\text { Silanpää, } \\
\text { Finland - } 1976\end{array}$ & $\begin{array}{l}\text { School- } \\
\text { children }\end{array}$ & 4825 & 7 & $37 \%$ & - \\
\hline Waters & Girls & - & $10-16$ & $93 \%$ & - \\
\hline $\begin{array}{l}\text { Bille } \\
\text { Sweden - } 1962\end{array}$ & - & - & 13 & $\begin{aligned} \text { male } & =58 \% \\
\text { female } & =59.3 \%\end{aligned}$ & - \\
\hline Andrasik et al & $\begin{array}{l}\text { School- } \\
\text { children }\end{array}$ & - & - & $99.7 \%$ & - \\
\hline $\begin{array}{l}\text { Mortimer et al. } \\
\text { United Kingdom } \\
1992\end{array}$ & Community & 317 & $9-11$ & $\begin{array}{c}\text { male }=78.4 \% \\
\text { female }=74.2 \%\end{array}$ & $\begin{array}{l}54.9 \% \text { male } \\
51.6 \% \text { female }\end{array}$ \\
\hline $\begin{array}{l}\text { Raieli et al. } \\
\text { Italy - } 1995\end{array}$ & $\begin{array}{l}\text { School- } \\
\text { children }\end{array}$ & 1445 & $11-14$ & $28 \%$ female & $19.9 \%$ male \\
\hline $\begin{array}{l}\text { Egermarck-Ericsson et al. } \\
\text { Sweden - } 1982\end{array}$ & $\begin{array}{l}\text { School- } \\
\text { children }\end{array}$ & 402 & $\begin{array}{l}7,11, \\
\text { and } 15\end{array}$ & $75 \%$ & - \\
\hline $\begin{array}{l}\text { Barea et al. } \\
\text { Brazil - } 1996\end{array}$ & $\begin{array}{l}\text { School- } \\
\text { children }\end{array}$ & 538 & $10-18$ & $\begin{array}{c}93.3 \% \\
\text { female }=94.4 \% \\
\text { male }=92.3 \%\end{array}$ & $\begin{array}{c}82.9 \% \\
\text { female }=87.9 \% \\
\text { male }=77.9 \%\end{array}$ \\
\hline
\end{tabular}

report ${ }^{9}$. Otherwise, problems of selection biases are less severe in pediatric headache due to the excellent school-based studies, because these settings provide reasonably representative samples of children living in an particular area ${ }^{11}$. For these reasons we preferred to conduct the present study in a school using questionnaires answered both by students and parents.

The similarity among the answers was very high in the two first questions. It allow us to say that in children of 9 or more years of age, the prevalence of headache may be determined by questionnaires answered by children, because in the present study it was observed that children and parental reports have the same value. It is important to mention that children and even parents are not obligated to understand terms such "migraine", "sinusitis" or "allergy", thus the results about the cause of headache in this study may be not so reliable because they were very dependent of previous knowledge by the subjects. Nevertheless, if we consider the great number of children and parents who marked the response "don't know", we can say that they probably have never looked for a medical assistance or that their headaches did not bring worrisome to them.

Our study observed that lifetime prevalence of headache was very high among the students analyzed. These results are comparable to those reported in Barea et al. study ${ }^{12}$, which found a lifetime prevalence of $93,2 \%$ in Brazilian schoolchildren, and to those reported by Rasmussen et $\mathrm{al}^{2}$, who stated that only $4 \%$ of the total population have never had a headache episode. Other studies also found a high prevalence of headache among students: $69 \%$ by age $14^{13,14}$; $93 \%$ of the girls of 10 to 16 years-old ${ }^{15}$; two thirds of children of 13 years of age ${ }^{16} ; 99.7 \% \%^{5} ; 56 \%$ of the boys and $55 \%$ of the girls ${ }^{17}$ (Table 6). 
The last year prevalence was about $90 \%$, as elevated as that found by Barea et al. ${ }^{12}$, which was $82,9 \%$. Mortimer et al. ${ }^{17}$ observed that the last 12 months prevalence among children of 9 to 11 years-old was $54.9 \%$ for boys and $51.6 \%$ for girls. Raielli et al. ${ }^{18}$, however, found a lower prevalence: $19.9 \%$ for boys and $28 \%$ for girls, both in the age group of 11 to 14 years-old (Table 6).

In our sample, the major part of the students had few headache episodes in the last year, and it was observed that girls have more frequent episodes than boys. These findings are in agreement with previous studies. According to Kristjánsdóttir and Wahlberg ${ }^{19}$, the headache prevalence is significantly higher among girls than boys. We found a higher number of children having rare headache episodes than having frequent ones in all ages. Egermarck-Ericksson ${ }^{20}$ reported that headache was more common in older (11 and 15 years of age) than in younger children, and more common in girls with 15 years of age than in boys of the same age. Similar results were reported by Bille ${ }^{16}$ in children of 10 to 15 years-old, as well as by Öster ${ }^{21}$. On the other hand, Bille ${ }^{16}$ did not observe significant differences in prevalence of headache considering sex in children of 7 years of age. Besides, Sillanpää ${ }^{6}$ reported that a frequency of less than once a month is as common in boys as in girls; however, higher frequencies are more common in girls. Most would argue that the gender difference is mainly due to hormonal changes; however, Deubner ${ }^{22}$ found no significant relationship between menarch status of the girls he studied and the presence of headache nor to headache severity, frequency or duration. Nevertheless, as stated by Kristjánsdóttir and Wahlberg ${ }^{19}$, the inconsistent differences found by gender indicate that other factors than gender and age might play a greater role in the prevalence of headache in children. It is important to emphasize that approximately $3 \%$ of the students had frequent headache episodes, and were not able to state the provoking factor. These children should be guided to look for a medical assistance.

Among the provoking factors, the most often reported were "flu" and "nervousness". It is in agreement with the results presented in a review of flow-sheet of children who sought consultations in a Brazilian Hospital complaining of headache: the most common diagnoses were upper airway infections $(26 \%)$, sinusitis $(15 \%)$, emotional factors $(15 \%)$, and visual disorders $(15 \%)^{3}$. In another study $^{20}$, the factors that provoked headache most often were "stress" (15\%), illness (14\%), and noisiness at school (13\%); $50 \%$ of the children with headache could not state any provoking factor. In our study, the misunderstanding about the causal factor was relatively high both for children and parents, but we did not found differences according to age (Table 5).

\section{CONCLUSIONS}

The results of the present study confirmed our first impression that would have a high prevalence of children suffering from headache in our environment. It gives emphasis to the important health problem that headache disorders represent among children.

When we compared questionnaires with information gave by children and parents, we could notice that, in general, there were not great differences between the results, particularly in the simplest questions, where "yes" or "no" were the only possible responses. So, we could say that when the objective of a epidemiologic study is to determine the prevalence of headache in children, both children and parental reports may be used.

\section{REFERENCES}

1. Duffner PK. Headaches in children. 48th Annual Meeting of the American Academy of Neurology, 1996.

2. Rasmussen BK, Jensen R, Schroll M, Olesen J. Epidemiology of headache in a general population: a prevalence study. J Clin Epidemiol 1991;44:1147-1157.

3. Rotta NT, Lago IS, Ohlweiler L, et al. Cefaléias como queixa ambulatorial em pediatria. Revista do HCPA 1985;5:25-27.

4. Kandt RS, Levine RM. Headache and acute illness in children. J Child Neurol 1987;2:22-27.

5. Andrasik F, Holroyd KA, Abell T. Prevalence of headache within a college student population: a preliminary analysis. Headache 1980;19:384-387.

6. Silanpää M. Prevalence of headache in prepuberty. Headache 1983;23:10-14. 
7. Linet MS, Stewart WF. Migraine headache: epidemiologic perspectives. Epidemiol Rev 1984;6:107-139.

8. Lipton RB, Silberstein SD, Stewart WF. An update on the epidemiology of migraine. Headache 1994;34:319-328.

9. Mindel JA, Andrasik F. Headache classification and factor analysis with a pediatric population. Headache 1987;27:96-101.

10. Stewart WF, Simon D, Schechter A, Lipton RB. Population variation in migraine prevalence: a meta-analysis. J Clin Epidemiol 1995;48:269-280.

11. Lipton RB. Classification and epidemiology of headaches in children. 48th Annual Meeting of the American Academy of Neurology, 1996.

12. Barea LM, Tannhauser M, Rotta NT. An epidemiologic study of headache among children and adolescents of southern Brazil. Cephalalgia 1996;16:545-549.

13. Sillanpää M. Prevalence of migraine and other headache in Finnish children starting school. Headache 1976;15:288-290.

14. Silanpää M. Changes in the prevalence of migraine and other headaches during the first seven school years. Headache 1983;23:15-19.

15. Waters WE. The epidemiology of migraine. Bracknell: Boehringer Ingelheim, 1974.

16. Bille B. Migraine in schoolchildren. Acta Paediatr Scand 1962;51:Suppl 136.

17. Mortimer MJ, Kay J, Jaron A. Epidemiology of headache and childhood migraine in an urban general practice using AD HOC, Vahlquist and IHS criteria. Dev Med Child Neurol 1992;34:1095-1101.

18. Raieli V, Raimondo D, Cammalleri R, Camarda R. Migraine headache in adolescents: a student population-based study in Monreale. Cephalalgia 1995;15:5-12.

19. Kristjánsdóttir G, Wahlberg V. Sociodemographic differences in the prevalence of self-reported headache in Icelandic schoolchildren. Headache 1993;33:376-380.

20. Egermarck-Eriksson I. Prevalence of headache in Swedish schoolchildren. Acta Paediatr Scand 1982;7:135-140.

21. Öster J. Recurrent abdominal pain, headache and limb pains in children and adolescents. Pediatrics 1972;50:429-436.

22. Deubner DC. An epidemiologic study of migraine and headache in 10-20 years-old. Headache 1977;17:173-180. 